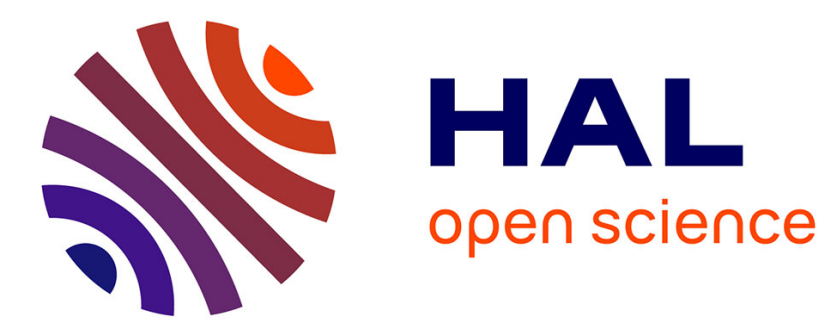

\title{
Introducing the concept ofmechanical texture in comminution: The case of concrete recycling
}

Florent Bourgeois, Nicholas Lippiatt, Malcolm S. Powell

\section{To cite this version:}

Florent Bourgeois, Nicholas Lippiatt, Malcolm S. Powell. Introducing the concept ofmechanical texture in comminution: The case of concrete recycling. International Journal of Mineral Processing, 2014, vol. 136, pp. 7-14. 10.1016/j.minpro.2014.09.012 . hal-01146906

\section{HAL Id: hal-01146906 https://hal.science/hal-01146906}

Submitted on 29 Apr 2015

HAL is a multi-disciplinary open access archive for the deposit and dissemination of scientific research documents, whether they are published or not. The documents may come from teaching and research institutions in France or abroad, or from public or private research centers.
L'archive ouverte pluridisciplinaire HAL, est destinée au dépôt et à la diffusion de documents scientifiques de niveau recherche, publiés ou non, émanant des établissements d'enseignement et de recherche français ou étrangers, des laboratoires publics ou privés. 


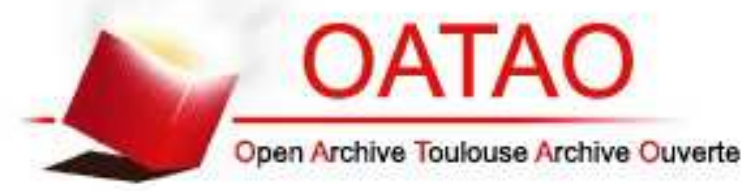

\section{Open Archive TOULOUSE Archive Ouverte (OATAO)}

OATAO is an open access repository that collects the work of Toulouse researchers and makes it freely available over the web where possible.

This is an author-deposited version published in : http://oatao.univ-toulouse.fr/ Eprints ID : 12851

To link to this article : doi: $10.1016 / \mathrm{j}$. minpro.2014.09.012

URL : http://dx.doi.org/10.1016/j.minpro.2014.09.012

To cite this version : Bourgeois, Florent and Lippiatt, Nicholas and Powell, Malcolm S. Introducing the concept ofmechanical texture in comminution: The case of concrete recycling. (2014) International Journal of Mineral Processing, vol. 136. pp. 7-14. ISSN 0301-7516

Any correspondance concerning this service should be sent to the repository administrator: staff-oatao@ listes-diff.inp-toulouse.fr 


\title{
Introducing the concept of mechanical texture in comminution: The case of concrete recycling
}

\author{
Florent S. Bourgeois ${ }^{a}$, Nicholas R. Lippiatt ${ }^{\mathrm{a}}$, Malcolm S. Powell ${ }^{\mathrm{b}}$ \\ a Laboratoire de Génie Chimique, UMR CNRS 5503, Université de Toulouse, France \\ b The University of Queensland, Sustainable Minerals Institute, Julius Kruttschnitt Mineral Research Centre, Australia
}

Keywords:

Mechanical texture

Comminution modeling

Ore breakage characterization

Concrete recycling

\begin{abstract}
A B S T R A C T
Modern comminution research and development are mainly product driven rather than material driven. An opinion that is gaining acceptance throughout the comminution community is that it is desirable for the comminution field to evolve toward material driven process design. To this end, this paper introduces the concept of mechanical texture, which corresponds to those textural properties of materials that have a direct bearing on their mechanical and fracture properties, which in turn should be the primary target for comminution process research and equipment design. The paper shows that mass specific fracture energy Ecs is a fracture parameter that is highly sensitive to variations in material texture, leading to selecting Ecs as a sound mechanical texture index. The paper then shows that, in the case of concrete, a set of specific features of the fracture porosity that can be measured inside concrete texture correlate highly with Ecs, thereby defining mechanical texture for concrete comminution. The demonstration that it is possible to establish a direct link between textural properties of concrete and macroscopic properties relevant to comminution shows that material driven comminution process modeling and design are possible and should be encouraged.
\end{abstract}

\section{Introduction}

Size reduction has been a pivotal process in the production of metals for as long as can be remembered, for beneficiation of minerals and waste. Size reduction unit operations are used throughout the minerals industry for the purpose of liberating valuable minerals, creating reactive surface area and producing desirable particle size distributions. As stated by Lynch in his introductory statement to his acclaimed 1977 textbook, "the extent to which breakage must proceed depends on the fineness of intergrowth or the "natural grain size" of the valuable particle. The natural grain size may vary widely..." (Lynch, 1977). This statement is perhaps one of the most important statements made in the early days of comminution modeling, as it recognizes the significance of ore texture in the size reduction process, and emphasizes its natural variability.

Surprisingly, despite the strong significance of material properties implied in Lynch's statement, the path which comminution research has followed since has by and large diverged away from the material to be processed. Neither has it been focusing on the process undergone by particles inside comminution unit operations, but has been dedicated to modeling and predicting the product output from the unit operations. This approach to comminution modeling and optimization has permitted formalization of a coherent and useful framework for what was prior an "empirical art", thus taking mineral comminution to an entirely new level. Over the past 4 decades, comminution research has served the industry well, giving it the means to increase production rates and meet society's needs. Comminution research did produce major conceptual advances, of which the most significant perhaps are the energy specific size reduction relationships, the breakage and selection functions for application of the population balance model to mineral comminution modeling, and the development of advanced simulation environments and control systems. And yet, the focus of comminution research and development has not been the ore itself, which finds itself embedded into sophisticated comminution models through some averaging property, distant from its actual physical properties and the variability thereof.

In accord with a number of researchers who have looked at the material contribution in comminution, such as Gaudin (1939), Schönert and Marktscheffel (1986) and Yashima et al. (1987), Powell et al. (2008) have formed the opinion that this comminution modeling approach has now reached an impasse, in that it can no longer evolve to meet the expectations of a modern mineral industry whose future depends on its ability to juggle scarcer and poorer ore bodies, rising energy costs, increasingly stringent environmental constraints, competition for access to water and fast changing societal needs. In order that the mineral industry can meet such a complex equation, Powell et al. propose a unified vision of comminution modeling, which some may consider as a paradigm shift relative to current practice. This vision focuses on the process itself, with the ambition of describing and predicting every individual event that occurs when comminuting an ore, down to the level of individual particles. This approach repositions the material to be processed to the heart of comminution modeling, which comes back to Lynch's statement cited above. This vision is largely fueled by the recent ability to simulate, with millions of objects, individual events that occur 
inside comminution machines in operation (Cleary, 2004, 2013; Cleary and Morrison, 2011; Weerasekara et al., 2013). In retrospect, it is fair to recognize that such capabilities were nonexistent and inconceivable when modern comminution modeling research emerged, which justifies the path taken by comminution research. Being able to simulate individual stress events inside a full scale comminution machine means, in turn, that physical properties of mineral particles relevant to comminution must be identified and modeled. Hence, relating mineral texture of a particle to the manner in which it fractures under stress is one critical issue in Powell et al.'s vision of the future of comminution modeling.

Prediction of the fragmentation behavior of a mineral particle under stress from knowledge of its texture is one of the key ingredients to Powell et al.'s vision of the future of comminution modeling. There are a number of steps to achieving this, of which the following 3 are perhaps the most significant:

Step 1 Measurement and modeling of the mineral texture, which is a spatial description of the components that make up the texture of the ore. Texture modeling for the purpose of enacting Powell et al.'s unified comminution model requires means for quantifying and reconstructing particle texture in three dimensions.

Step 2 Identification of the textural components responsible for the mechanical/fracture behavior of the texture under stress, whose combination define what is here referred to as mechanical texture. The concept of mechanical texture embodies the direct link between mineral texture and the mechanical/fracture behavior of the ore under stress.

Step 3 Simulation of the fracture of a mineral particle with known mechanical texture under given loading conditions (Weatherley, 2013). This 3rd step provides the link between ore texture and DEM modeling for predicting the outcome of comminution processes.

The present contribution focuses on the notion of mechanical texture, which is the pivot between mineral texture and DEM modeling.

\section{Definition of mechanical texture}

As stated above, assigning a mechanical texture to an ore implies that one identifies and ranks the textural features responsible for the physical fragmentation behavior of the texture of interest under stress, and the variability thereof. The set of textural features that govern the mechanical behavior of the material of interest defines the mechanical texture of the material. Textural features of interest may be associated with grain boundaries, pores, hard inclusions, shape, etc. This raises the question of how one may identify such textural properties in the first place.

The idea proposed here is to define some simple scheme for identifying such textural properties. When fracturing single particles by impact on a Hopkinson bar, one recognizes that individual particles behave differently from one another. The range of mechanical behavior of the particles is a direct measure of the variability of the mechanical texture of the material. We propose here that identification of mechanical texture components of significance relies on identifying those components which correlate most with the variability of mechanical behavior measured by sensitive equipment such as Hopkinson bars. Slow compression testing equipment could also be used to assess mechanical fracture properties of brittle particles, the rate of energy input being one decisive factor as to the choice of one method over the other.

This scheme first requires that one identifies the macroscopic fracture property which varies most significantly for a given lot of particles, its variability being taken as an indicator that the property in question best captures the intrinsic mechanical heterogeneity of the material of interest. This property is a macroscopic index for the mechanical texture of the ore. Identification of textural properties which comprise the mechanical texture of the material of interest will be those which correlate most with this macroscopic index.
In this section, we establish that mass specific fracture energy, noted Ecs, as measured by Hopkinson bar impact tests is a sound macroscopic index of mechanical heterogeneity. It is also interesting to note that Ecs has become a parameter of convergence for all current comminution models, which makes it likely that the concept of mechanical texture should eventually interface well with current comminution modeling schemes.

To derive this index, to which we shall eventually correlate the textural properties of the ore, the authors decided to test what may be considered a model material due to its extreme level of homogeneity from a mineral texture standpoint. Six millimeter soda lime glass beads underwent impact testing on a Hopkinson bar (Bourgeois and Banini, 2002).

Fig. 1 gives the force-time profiles measured by Hopkinson bar impact testing for the $6 \mathrm{~mm}$ soda lime glass beads. Tests were conducted using a $60 \mathrm{~mm} / 882 \mathrm{~g}$ stainless steel ball bearing dropped from $80 \mathrm{~mm}$. The clean superimposition of the force rise, which is expected given that the particles have the same shape, confirms the repeatability of the test protocol. What is significant here is the range of fracture forces measured for individual particles. This range spans from $2231 \mathrm{~N}$ to $4253 \mathrm{~N}$, i.e. it varies by a factor of 2 , which can be described satisfactorily using a Weibull distribution with parameters $k=6.6$ and $\lambda=3433 \mathrm{~N}$. The mass specific fracture energy Ecs, which is the energy actually absorbed by the particle before the point of fracture-not to be mistaken for the potential energy of the striker-varies by a factor of 4.5. The broad range of mass specific fracture energies reflects the significant variability of mechanical properties of the soda lime glass beads, despite their being a model material.

Force-time measurement from Hopkinson bar tests yields estimation of particle strength, stiffness and mass specific fracture energy (Bourgeois, 1993; Tavares and King, 1998). The former relates to the maximum load which the particle can sustain before fracturing, whereas the latter is associated to the amount of deformation of the loaded particle. Distributions of force at fracture and mass specific fracture energy measured for soda lime glass beads are shown in Fig. 2.

The distribution of fracture properties, which is revealed by the Hopkinson impact test carried out on individual particles, yields a good appreciation of how significant the distribution of mechanical properties is, even for a material seemingly as texturally homogeneous as soda lime glass. Tavares and King (1998) have shown that there is a strong correlation between particle strength and mass specific fracture energy for glass beads, but the issue here is not so much about the correlation between mean values, but more on the variability, i.e. the spread of the distribution of the measured properties.

The measured values of relative standard deviation (RSD) for mass specific fracture energy, strength and stiffness for the $6 \mathrm{~mm}$ soda lime glass beads were $0.39,0.17$ and 0.16 respectively.

Of the 3 properties which are readily available from Hopkinson bar impact tests, the mass specific fracture energy exhibits the largest variability. It is concluded that the mass specific fracture energy is a macroscopic

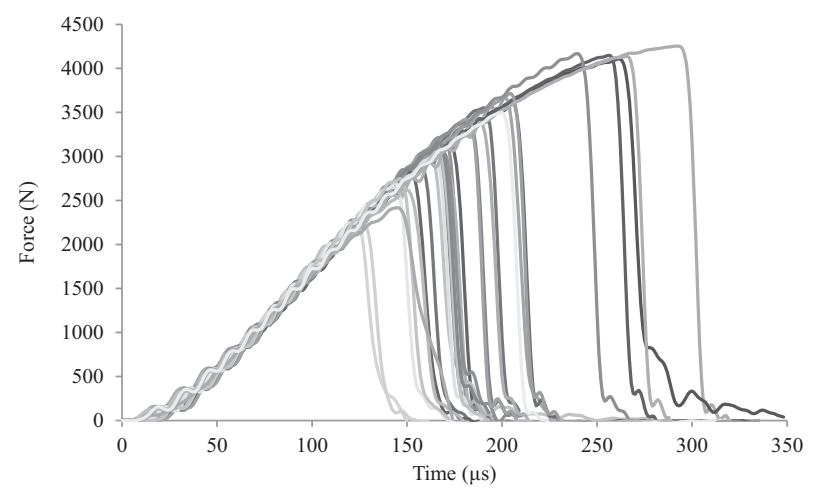

Fig. 1. Force-time profiles measured on 31 soda lime glass beads. 

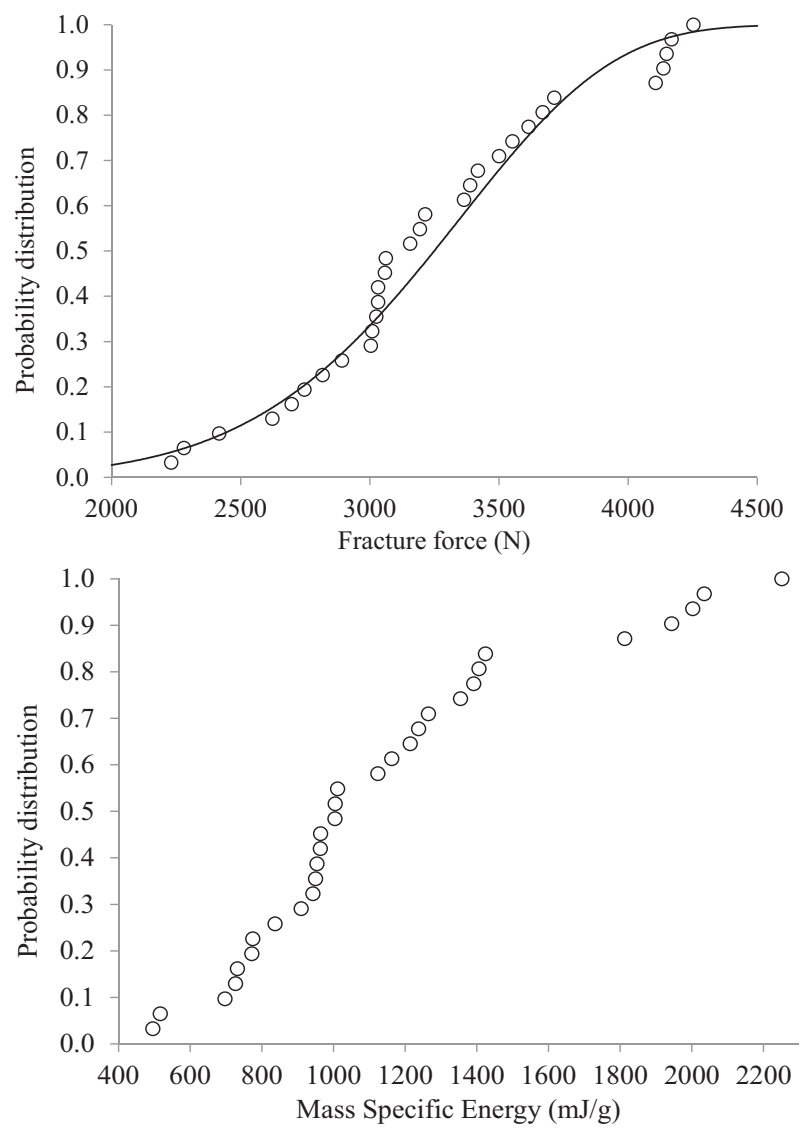

Fig. 2. Measured distributions of force at fracture and the mass specific fracture energy for $6 \mathrm{~mm}$ soda lime glass beads.

index most sensitive to the variability in impact fracture behavior of the soda lime glass beads; hence textural properties that correlate strongly with Ecs should define the mechanical texture of the glass beads.

Taking numerous sources of Hopkinson bar test results from the literature for samples of 25 particles or more, independently obtained on a wide variety of materials, natural ores and manmade materials, Fig. 3 shows the RSD for mass specific fracture energy and strength. By and large, the majority of points are located near or above the bisecting line. Indeed, the RSD of the mass specific fracture energy is, on average,

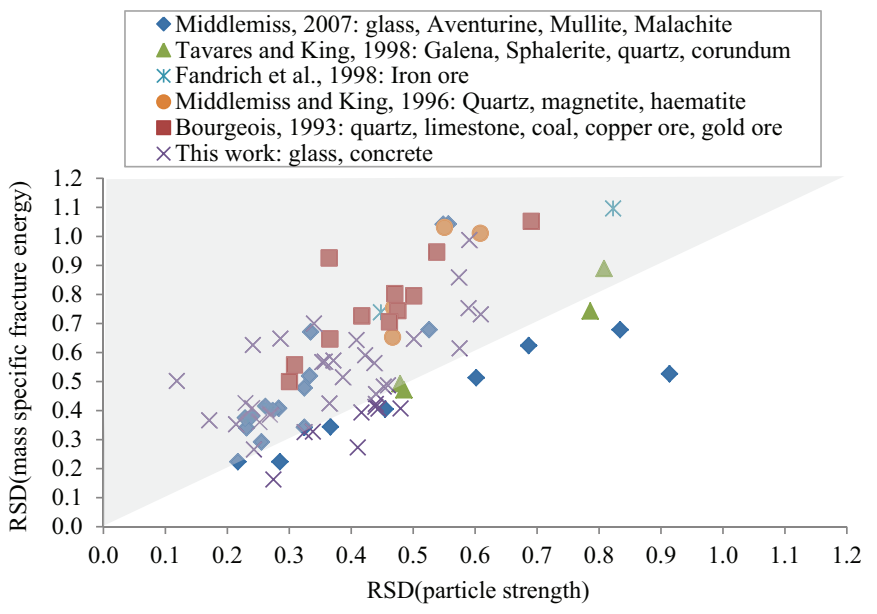

Fig. 3. Validation of the Ecs as the Hopkinson bar test derived fracture property most sensitive to variations in mechanical texture (Frandrich et al., 1998; Middlemiss and King, 1996).
$48 \%$ higher than that of particle strength. It is concluded that mass specific fracture energy is an acceptable index for the variability of fracture properties of impact loaded materials.

From this analysis of mechanical texture variability from Hopkinson bar testing, it is proposed to use mass specific fracture energy as the mechanical texture index of the ore. The RSD of the Ecs therefore is a macroscopic measure of the variability in mechanical texture of the material.

Using this index requires that a large enough number of particles be tested with the Hopkinson bar. Analysis of textural heterogeneity from an insufficient number of particles would be futile, given the wide confidence intervals for the estimate of the standard deviation. In practical terms, the analysis proposed here requires that 30 particles or more be tested, as it is commonly applied to Hopkinson bar testing.

For the $6 \mathrm{~mm}$ soda lime beads, $E c s=292.0 \mathrm{~mJ} / \mathrm{g}$ and $\operatorname{RSD}(E C S)=\frac{114.1 \mathrm{~mJ} / \mathrm{g}}{292.0 \mathrm{~mJ} / \mathrm{g}}=0.4$

For a given material, it is known that Ecs obeys a log-normal distribution (Bourgeois, 1993; Middlemiss, 2007). Fig. 4 shows the distribution of $\operatorname{RSD}(E c s)$ values from the data plotted in Fig. 3. The distribution can be approximately described by a left-truncated Gaussian distribution with mean $=0.56$ and standard deviation $=0.23$.

Testing an ore on the Hopkinson bar and positioning its mechanical texture variability index $\operatorname{RSD}(E c s)$ in this distribution may be a useful tool for ranking its heterogeneity in mechanical texture against known materials. It is also an interesting manner by which one may readily quantify the effect of a pretreatment on the mechanical properties of ores. Indeed, pretreatment is expected to affect the mechanical texture of the ore, both in mean value and variation about the mean, the former being captured by the proposed mechanical texture index Ecs and the latter by $\operatorname{RSD}(E c s)$.

The distribution of Ecs and that of $\mathrm{RSD}$ (Ecs) are statistical fracture properties which can be fed into population balance models (King and Bourgeois, 1993). However, they are not relevant for DEM comminution models which require a model mechanical texture at the particle scale. As a step forward toward such an endeavor, the next section of the paper investigates the textural features responsible for the mechanical texture, which is defined on a macroscopic scale by Ecs. Such textural properties are those which correlate most with the proposed mechanical texture variability index Ecs and associated variability $\operatorname{RSD}(E c s)$. Provided that such properties can be identified, they will in turn need to be modeled spatially, which reverts to the mineral texture modeling step presented in introduction, in order to serve as input to DEM modeling.

\section{Unraveling the link between mineral and mechanical texture}

As indicated in the introductory statements, material driven process design requires identification of those textural properties that dictate

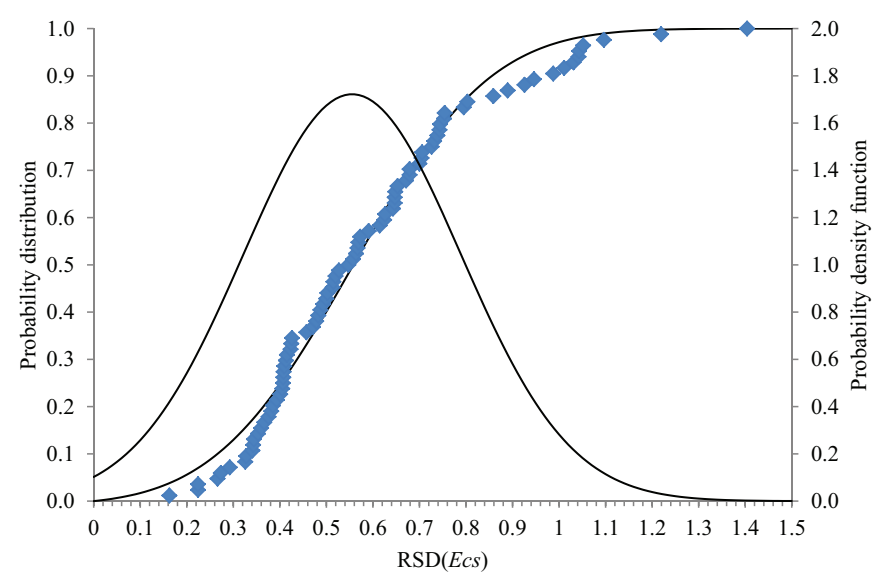

Fig. 4. Distribution of $\mathrm{RSD}(E c s)$ for the data from Fig. 3. 
the mechanical texture of the material. Such properties are utterly material specific; hence it is difficult to imagine some universal way to link mineral and mechanical textures. Nevertheless, the link exists, and it ought to be the focus of material driven process design. The following section explores this link for the case of concrete, with the end objective of designing a material driven process for recycling concrete. In the context of concrete recycling, processability is measured in terms of aggregate liberation, comminution product fineness and mechanical strength of the material. For the purpose of exploring the link between texture and processability, the paper focuses on concrete strength as an example, which, in processing terms, equates to comminution energy. The question therefore is to identify the textural parameters that make up the mechanical texture of concrete, i.e. the textural parameters which correlate most strongly with the proposed mechanical texture index Ecs.

The textural property which is considered to be of the most interest is related to porosity. Porosity is a complex textural property in concrete, which can be manipulated in a number of ways. It can be changed at the mixing stage, through varying the water to cement ratio for example, but also by heating. In order to generate different fracture porosity patterns, the properties of which will be correlated to the mechanical texture variability index, the authors chose to heat laboratory-made concrete samples either externally, with an oven, or using microwaves (Lippiatt and Bourgeois, 2012).

\subsection{Materials and methods}

Concrete samples were cast from a mixture of CEM II/B-LL 32.5N Portland cement and narrowly sized $2-2.5 \mathrm{~mm}$ siliceous aggregate particles. Five different concretes were made using three different water to cement ratios $(\mathrm{w} / \mathrm{c})$ and three different aggregate to cement ratios $(\mathrm{a} / \mathrm{c})$. The sample preparation and testing protocol are represented in Fig. 5.

Samples were cast in $20 \mathrm{~mm}(\approx 10 \mathrm{~g})$ cylinders and were tested in two ways. The first protocol consisted of heating whole cylindrical particles, and then subjecting them to Hopkinson bar impact testing. The second protocol, dedicated to texture analysis, consisted of diamond saw cutting samples to expose a flat surface in the cylindrical samples prior to heating them, and then observing the flat surface by electron microscopy (SEM) after treatment. The primary concern of this protocol was avoiding tampering with the treated sample surface before observation. Porosity was characterized via a number of features measured on SEM digital images. Approximately 20 images were used per sample, of which 10 were taken at a magnification of $40 \times$ and 10 at a magnification of $200 \times$, yielding images with resolutions of $9 \mu \mathrm{m} /$ pixel and $1.8 \mu \mathrm{m} /$ pixel respectively. For full details, see Lippiatt and Bourgeois (2014).

\subsection{Texture and embrittlement}

On a macroscopic scale, an increase in the porosity of concrete due to heating causes concrete to weaken. This is a key problem associated with the fire resistance of concrete (Willam et al., 2005). Fig. 6 shows the mass specific fracture energy obtained for a set of 33 concrete samples with varying aggregate to cement ratios $(\mathrm{a} / \mathrm{c})$. Mass specific fracture energy, as measured by Hopkinson bar testing, exhibits an inverse relationship with total porosity, as measured by mercury intrusion porosimetry (MIP). The correlation is significant, and yet the measurements show a large degree of scattering.

Fig. 6 confirms a direct link between textural (porosity) and mechanical (Ecs) properties for concrete. However the macroscopic nature of total intrusion porosity means that it has little power in explaining the fracture behavior, hence the scattering of Fig. 6. It is therefore not an appropriate property to be used as a mechanical texture property. The source of changes in mechanical texture, as measured by Ecs, was sought at the local scale by quantifying the properties of fracture porosity. Indeed, defining the term fracture porosity to describe the fracture system in concrete, the authors have shown (Lippiatt and Bourgeois, 2014) that distinct changes in fracture porosity occur, albeit at different scales inside the concrete, during heating. Through observation of numerous heated concrete samples, the authors have identified that fracture porosity, which changes with heating temperature and duration of exposure to heat, occurs as two distinct fracture networks. Fig. 7 illustrates the form taken by fracture porosity inside a concrete sample.

The first system of fractures consists of large scale fractures that run along grain boundaries, often joining aggregate particles and air pockets present in the cement phase. The formation of this primary network, noted N1, is associated with the aggregate-cement interfacial transition zone or ITZ (Mehta and Monteiro, 2001), which is a few tens of micrometers thick. It is known for its high relative water content, steep moisture gradient, high porosity and high portlandite content. Air pockets play a role in dissipating heat-induced stresses inside the cement matrix; hence the convergence of primary network fractures as seen in Fig. 7.

Observation of concrete texture for low microwave energy inputs and low furnace temperature points toward fractures being initiated near aggregate grain boundaries, hence inside the ITZ and forming a more or less connected network of large fractures that percolates through the concrete sample. X-ray tomographic images confirm the existence and percolation of the primary fracture network.

For the purpose of analysis primary network is defined as all fractures at the cement paste/aggregate interface and all fractures they are connected to. In the case of divergence only the widest connection was included in the primary network. In the case of two branches of equal width only the longest was included in the primary network. All unaccounted fractures in the cement paste were defined as belonging the secondary network. All analysis of the primary network was performed

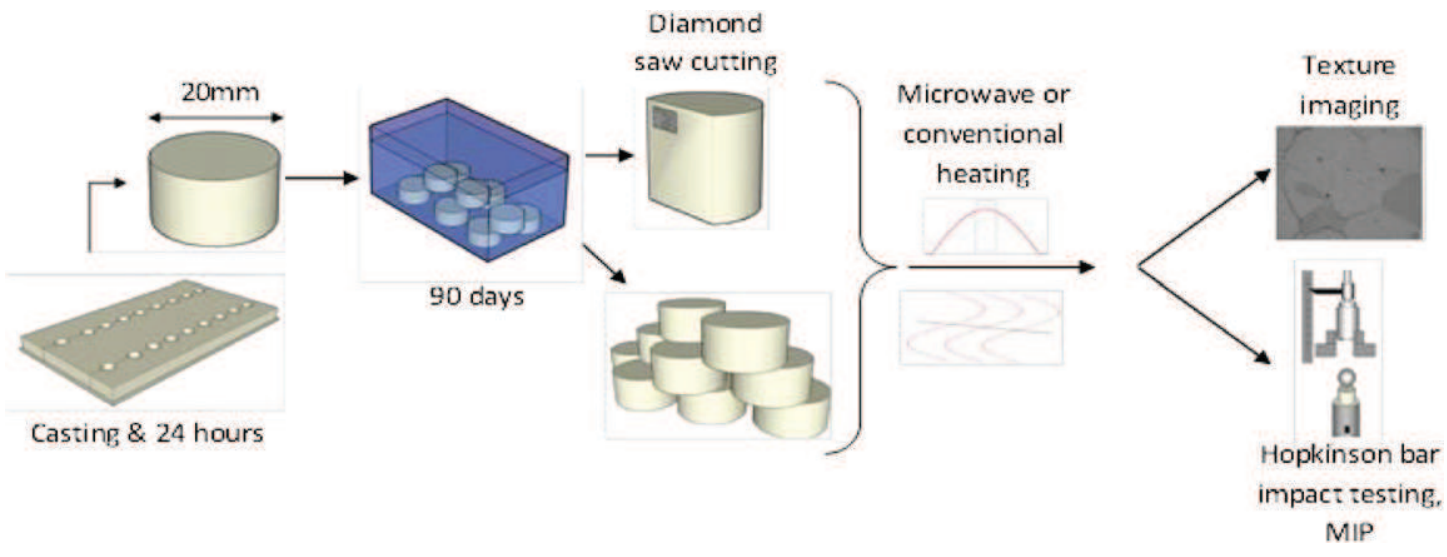

Fig. 5. Concrete sample preparation and testing protocols. 


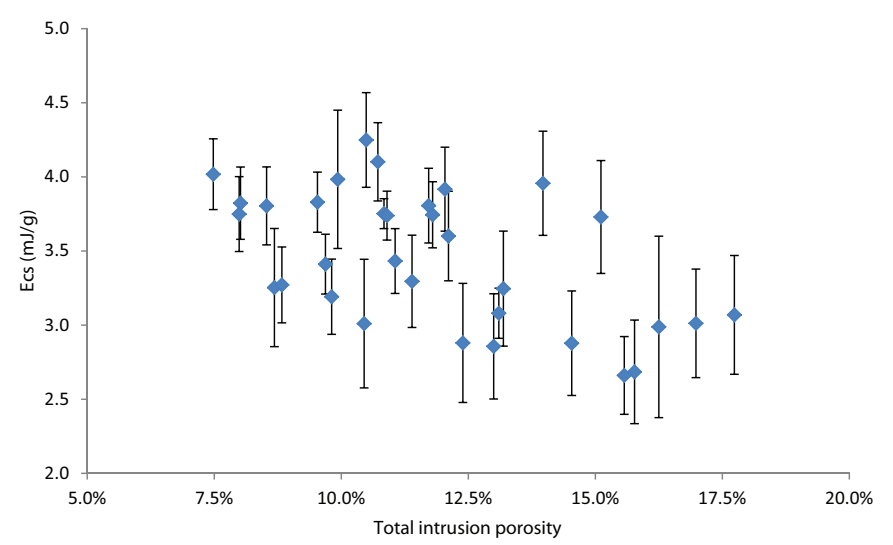

Fig. 6. Measured variation between mass specific fracture energy and total porosity measured by MIP.

using images at $40 \times$ magnification ( $9 \mu \mathrm{m}$ per pixel). Analysis of the secondary network was performed using images at $200 \times$ magnification (1.8 $\mu \mathrm{m}$ per pixel). For further details see Lippiatt and Bourgeois (2014). To understand the textural properties used in analysis first requires an understanding of the division of image areas. Aggregate particles and cement paste could be easily identified so pixels were divided into those that showed part of an aggregate particle and those that did not, the latter defined as cement area for analysis purposes.

The properties which define the primary fracture network are:

- Total fracture length $\left(\mathrm{m} / \mathrm{m}^{2}\right)$ : The sum of the lengths of all the primary network fractures in all highlighted images divided by the cement area in all highlighted images for that combination of concrete type and pre-treatment.

- Fracture area $\left(\mathrm{m}^{2} / \mathrm{m}^{2}\right)$ : The sum of the area of all the primary network fractures in all highlighted images divided by the cement area in all highlighted images for that combination of concrete type and pre-treatment.

- Textural liberation: The ratio of the total length of fractures at the aggregate/cement interface divided by the total length of aggregate/ cement interface.

- Nodes per object: How many fractures diverged from the aggregate/ cement paste interface into the cement paste phase.

The secondary fracture category consists of a large number of small fractures that seem to form randomly, or at least more randomly than fractures in the primary network, throughout the cement paste phase. The density of fractures in this secondary network, noted N2, increases with heat energy input.
Total fracture length and fracture area were defined in the same way for both the primary and secondary network. The other properties which define the secondary fracture network are:

- Number of branches per object: The average number of fracture intervals (branches) that diverge from every grouping of connected fractures (objects).

- Number of branches per fracture area: Using the same number of branches as the definition above but dividing the value by cement area rather than number of objects.

- Euler number: A measure of the connectivity of the fracture network using the Euler characteristic relating the number of vertices $(\mathrm{V})$, edges (E) and faces (F) (Early, 1999).

$x=V-E+F$

which for this case becomes

$x=V-b+1$

where $\mathrm{b}$ is the number of branches, $\mathrm{as} F=1$ for a two dimensional object.

As it was discussed in the introductory statement, mass specific fracture energy is a macroscopic material property which appears to be most sensitive to textural variation in the material, hence the present focus on chasing the textural parameters responsible for mass specific fracture energy in concrete. This endeavor however should be understood as a specific illustration of the concept of finding causal relationship between textural properties and macroscopic properties of materials relevant from a processing perspective. In this paper, textural properties of concern are those of the primary and secondary fracture networks, whereas the physical fracture property of processing value (and changes thereof) is the mass specific fracture energy.

The principal component analysis (PCA) was selected for identification of causal relationships between properties of fracture porosity, mass specific fracture energy Ecs and $\operatorname{RSD}(E c s)$. Principal components were calculated using textural variables from the primary and secondary fracture networks only. The mass specific fracture energy and its RSD, measured by Hopkinson bar impact testing, were added to the PCA only after the principal components had already been calculated relative to the textural variables. The rationale behind defining the principal components solely in terms of textural parameters first and then adding the illustrative mechanical properties afterwards was deemed suitable for finding the textural properties that make up the mechanical texture of concrete. This approach is in fact implied by the concept of mechanical texture.

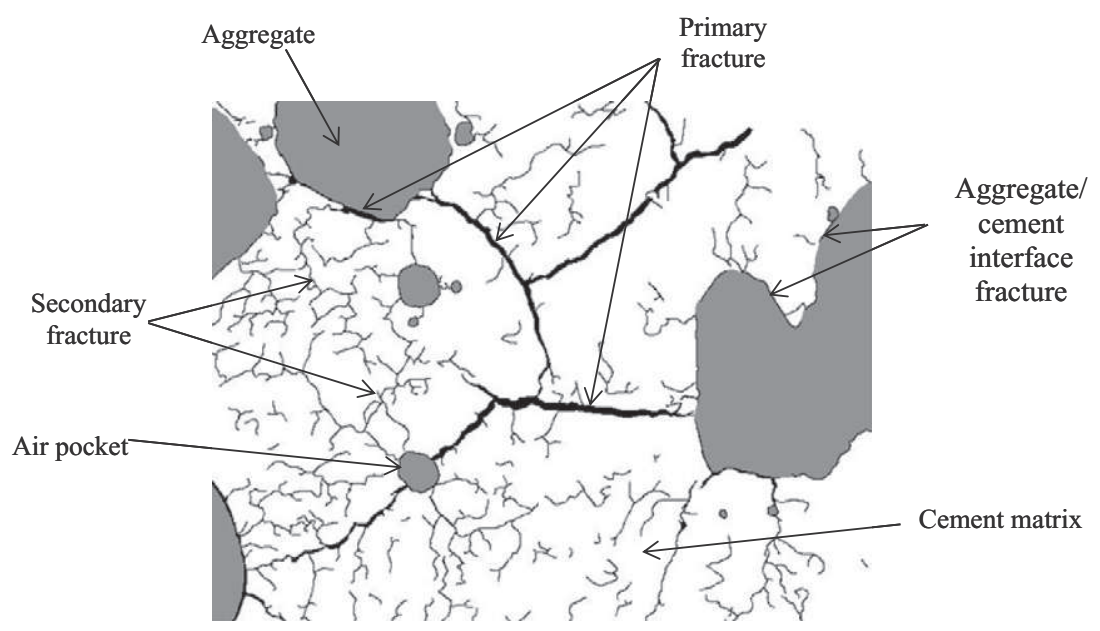

Fig. 7. SEM photomicrograph exemplifying the formation of heat-induced primary and secondary fracture networks. 
This PCA analysis used:

- 4 textural variables for the primary fracture network N1 (N1_var5 to N1_var8), and 5 textural variables for the secondary fracture network N2 (N2_var9 to N2_var13), so that the PCA analysis was carried out using 9 variables. Table 1 gives the correspondence between the PCA variables and their actual physical meaning.

- The number of individuals was 34.

- The illustrative variables were the mass specific fracture energy Ecs $(\mathrm{mJ} / \mathrm{g})$ and $\mathrm{RSD}(E c s)$. Since this Ecs is log-normally distributed, the variable used in the PCA analysis was the natural logarithm $\ln (E c S)$, which is normally distributed. It was checked (see Fig. 4) that $\mathrm{RSD}(E c S)$ is normally distributed; hence, it was used directly.

After normalization, the normal distribution of all 9 textural variables was verified. Fig. 8 shows the first two components of the PCA analysis run using the 9 textural parameters of the fracture networks. Principal components 1 and 2 accounted for $58 \%$ and $20 \%$ of the total variation respectively. These components are noted PC1 and PC2 respectively.

On the circle of correlations, we firstly note that there are two clearly separated clusters, each in a separate quadrant, corresponding to the primary and secondary fracture networks. This lends credence to the assertion made from visual observations that there are two distinct systems of fractures occurring in concrete. Secondly, we observe that the primary and secondary clusters are both on the same side of the first principal component (noted PC1) axis, so they are highly correlated. This is expected as we do not observe the formation of the N2 network without the N1 network being present.

Positioning the 34 individuals used to carry out the PCA analysis inside the PC1-PC2 plane, as shown in Fig. 9, we find that:

- All unheated concrete samples, ending with the " $r$ " subscript, stand clustered and close to the left side of the PC1 axis, whereas the concrete sample with the least strength, which was obtained by heating to $500{ }^{\circ} \mathrm{C}$ in a furnace, is directly opposite the PC1 axis. From these observations, it is inferred that the PC1 axis is a measure of the degree of fracturing of the texture.

- Defining the N2/N1 fracture length ratio as the ratio between the total fracture length of the $\mathrm{N} 2$ and $\mathrm{N} 1$ fracture networks, we observe that the individuals whose ratio is highest stand on the lower part of the PC1-PC2 plane, close to the PC2 axis, whereas samples with the lowest ratios stand directly opposite. The conclusion is that the PC2 axis is a measure of the degree of significance of the secondary fracture network over the primary fracture network.

Having given physical meaning to the first 2 principal components, the illustrative variables $\ln (E c s)$ and $\operatorname{RSD}(E c s)$ were then placed onto the circle of correlations. The result is shown in Fig. 10.

We observe that:

- The Ecs property (plotted as $\ln (E c s)$ for the sake of normality) sits very close to the PC1 axis, opposite to that of the N1 and N2 variables. This means, as one would expect, that the denser the fracture networks, the lesser the mean mass specific fracture energy of

\section{Table 1}

Correspondence table between PCA variables and their actual physical meaning.

\begin{tabular}{lll}
\hline Fracture network & Texture variable name & Texture variable definition \\
\hline Primary & N1_var5 & Total fracture length $\left(\mathrm{m} / \mathrm{m}^{2}\right)$ \\
& N1_var6 & Fracture area $\left(\mathrm{m}^{2} / \mathrm{m}^{2}\right)$ \\
& N1_var7 & Textural liberation \\
& N1_var8 & Nodes per object \\
Secondary & N2_var9 & Total fracture length $\left(\mathrm{m} / \mathrm{m}^{2}\right)$ \\
& N2_var10 & Fracture area $\left(\mathrm{m}^{2} / \mathrm{m}^{2}\right)$ \\
& N2_var11 & Number of branches per fracture \\
& N2_var12 & Number of branches per fracture area \\
& N2_var13 & Euler-Poincaré number \\
\hline
\end{tabular}

concrete. This result is a clear evidence of the direct link between texture and fracture properties in concrete.

- We observe that $\mathrm{RSD}(E C S)$ on the right hand side of the PC1 axis. This indicates that for concrete, the variability in mass specific fracture energy is associated with the extent of the fracture networks. However, the closeness of RSD(Ecs) to the PC1 axis means that the variability of Ecs increases with fracture porosity, irrespective of the type of fractures

As an increase in Ecs impacts comminution efficiency negatively from an energy utilization point of view, this analysis indicates that the production of a primary network dominant fracture is one key to effectively reducing the mass specific fracture energy of concrete. This result establishes a direct link between concrete's mechanical texture and processing performance criteria.

From analysis of correlation between properties of the fracture porosity and the mass specific fracture energy in concrete, it so happens that both fracture networks contribute to defining the mechanical texture of concrete. While the extent of both networks appears to govern the mean value of the mass specific fracture energy, whereas the relative dominance of secondary fracture network controls its variability.

\section{Implications for comminution modeling and processing of concrete}

Mechanical texture was defined here as the subset of textural properties that govern the mechanical and fracture behavior of particles. Such properties need to be identified, modeled and then fed into DEM comminution models. Having selected mass specific fracture energy as a macroscopic material fracture property of interest, the work established a causal relationship with fracture porosity, a local textural property, in the case of concrete. With concrete, one textural property which is associated with mechanical texture is therefore fracture porosity. This is not saying that other textural properties are not of significance; however additional research is necessary to establish additional correlations between local textural parameters and mechanical properties of concrete.

Mass specific fracture energy however embeds one aspect of comminution, the others being the size distribution of the progeny fragments and the liberation in the case of multiphase materials. There exists a significant body of literature on comminution and liberation of concrete (Jeschke et al., 2006; Khanal et al., 2005). As with Ecs, the authors have identified direct links between the fracture porosity in concrete and the size distribution of progeny particles. Links between concrete textural properties and liberation of aggregates from cement paste have also been established. These links are not described here for the sake of brevity; however, they add to the value of applying the concept of mechanical texture in comminution modeling and analysis.

Definition of mechanical texture however is one step, albeit critical, to feeding material properties into DEM comminution models. The next step consists in deriving a model of the mechanical texture that can be used to simulate the mechanical texture of particles, so that it can be used in DEM simulation environments. This mechanical texture simulation step is a complex issue which requires, in the case of concrete, a spatial description of fracture porosity, in addition to describing the spatial distribution of aggregate particles (Qian and Schlange, 2013).

Identification of causal pathways between texture and physical fracture property also has direct implications for processing, whether for concrete or any other material subjected to processing for beneficiation. The recognition that fracture porosity variables correlate with the mass specific fracture energy and its RSD for concrete has direct bearing on designing a material driven recycling process. Indeed, processes applied to concrete should be investigated in relation to their ability to alter the growth of both primary and secondary fracture networks in concrete, which to the authors' knowledge has not yet been undertaken.

In the case of concrete, the link that was established between the primary fracture network and the mass specific fracture energy indicates that a process which induces an N1-like fracture porosity will be best 


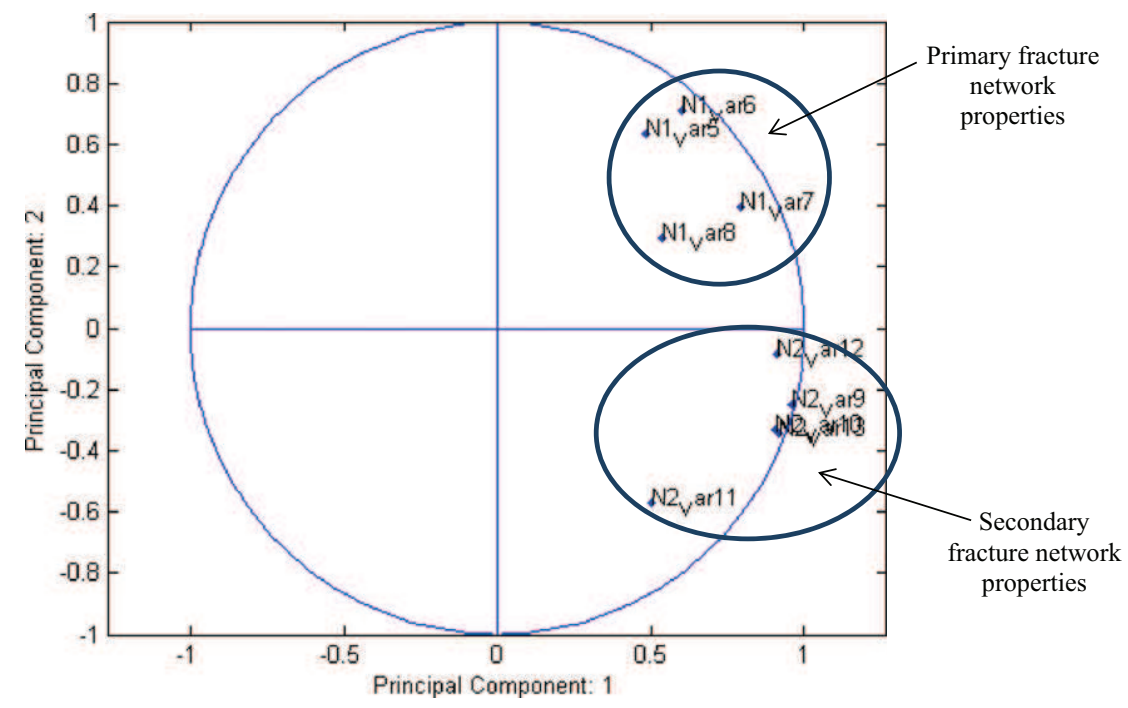

Fig. 8. PCA analysis results for primary and secondary fracture network properties.

suited for reducing the mass specific fracture energy of concrete; hence, it will be most efficient for minimizing comminution energy. On the other hand, a process that generates an N2-like fracture porosity will yield greater variability in mass specific fracture energy, which may not be desirable from a processing viewpoint.

When microwave energy is applied to concrete samples, the authors have found that low microwave energy input is sufficient to generate the greatest change in primary network growth, whereas longer exposure to microwave yields development of the secondary fracture network. Processing wise, this means that application of low microwave energy is sufficient for weakening concrete, as it targets the textural property most responsible for the mass specific fracture energy of concrete.

Should other processing criteria be used, such as the physical liberation of aggregate or the fineness of comminuted concrete, other textural properties may intervene as additional components of mechanical texture, and hence should be additional processing targets in addition to additional inputs to be considered in texture simulation for DEM modeling. Deriving causal relationships between textural and macroscopic properties, as confirmed through the concrete example used in relation to mechanical texture, as well as embedding them in texture models for
DEM simulation, is expected to show new paths for designing efficient and material specific processing solutions, and for adding realistic fracture models into DEM comminution models.

\section{Conclusions}

Design of comminution processes for ores and wastes is largely product driven. It is postulated that more efficient processes could be designed and operated should they be driven by material properties instead. This approach, which operates a significant shift in the way one may approach comminution modeling and equipment design should target the set of textural properties that control the fracture properties of the ore. This set of textural properties is here within defined as mechanical texture.

Through investigation of soda lime glass beads, a model material from the textural standpoint, combined with a large number of published data from the literature, this work justified using the mass specific fracture energy Ecs as a sound macroscopic index for mechanical texture. This result was established on the basis that the relative standard deviation of Ecs exhibited the highest value among Hopkinson

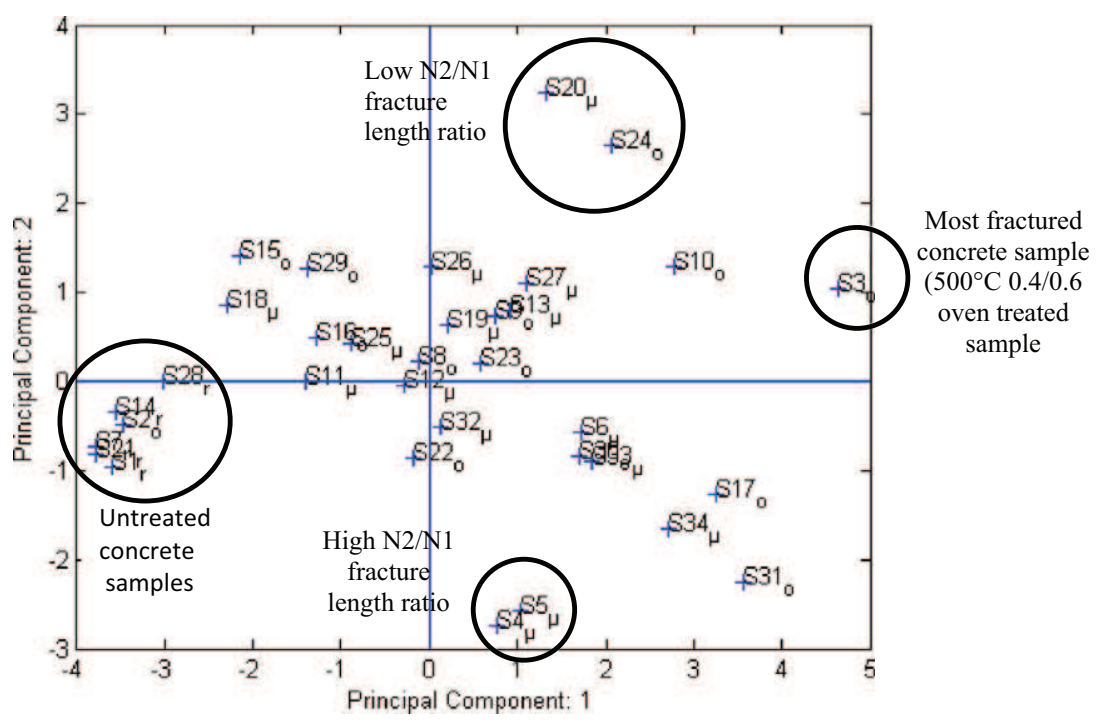

Fig. 9. Individuals plot, confirming that the 1 st principal component axis is a measure of the level of fracturing of the concrete sample, whereas the 2 nd principal component axis is a measure of the dominance of the secondary fracture network. 


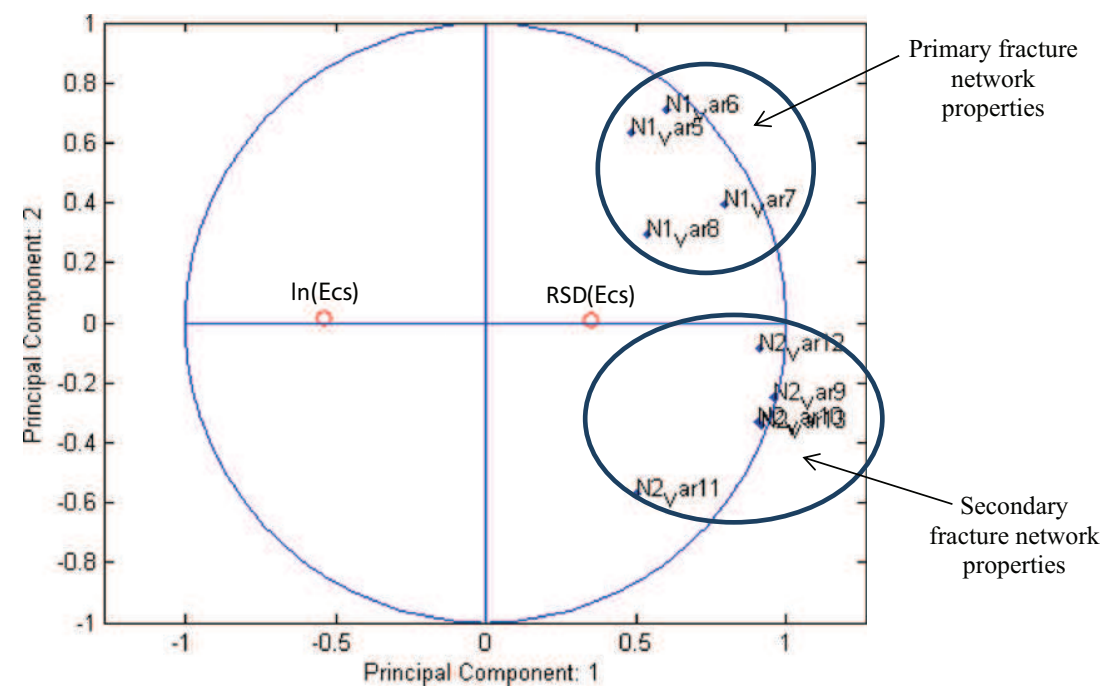

Fig. 10. Positioning the illustrative variables $\ln (E c S)$ and $\operatorname{RSD}(E c S)$ on the circle of correlations.

bar single-particle impact test measured properties, directing our choosing Ecs as most sensitive to variability in texture as it relates to the comminution behavior of the ore.

Relating textural properties of a material to Ecs is very much material dependent. Using concrete as an example, textural properties associated with fracture porosity were convincingly correlated through principal component analysis to Ecs. These textural properties included properties of what the authors have identified as the primary and secondary fracture networks, which describe the fracture system in concrete. It was therefore concluded that these fracture networks define the concept of mechanical texture as it applies to concrete.

For process design, the implication of mechanical texture, as quantified in the case of concrete, was that processes capable of targeting changes in the primary fracture network are desirable for low energy comminution of concrete. The authors found that low energy microwave heating is a process which favors development of the primary fracture network. Other textural variables however may need to be taken into account in the definition of concrete's mechanical texture when considering aggregate liberation and product fineness, so that the information gathered here about fracture porosity networks may not be sufficient to define the terms of reference of a material-based processing scheme for concrete.

\section{Acknowledgments}

The authors acknowledge the financial support of the Agence Nationale de la Recherche (the French National Research Agency) through the COFRAGE project from the ANR-Ecotech 2009 research program.

\section{References}

Bourgeois, F., 1993. Single-particle fracture as a basis for microscale modeling of comminution processes(Ph.D. dissertation) Department of Metallurgical Engineering, The University of Utah.

Bourgeois, F., Banini, G., 2002. A portable load cell for in-situ ore impact breakage testing. Int. J. Miner. Process. 65, 31-54.

Cleary, P.W., 2004. Large scale industrial DEM modelling. Eng. Comput. 21, 169-204.

Cleary, P., 2013. Particle scale modelling of breakage and transport in comminution devices. In: Kwade, A., Breitung-Faes, S., Steiner, D. (Eds.), Proceedings of the 13th European
Symposium on Comminution \& Classification, pp. 39-43 (9th-12th September 2013 , Braunschweig, Germany).

Cleary, P.W., Morrison, R.D., 2011. Understanding fine ore breakage in a laboratory scale ball mill using DEM. Miner. Eng. 24, 352-366.

Early, E., 1999. On the Euler characteristic. MIT Undergr. J. Math. 1, 37-48.

Frandrich, R., Clout, J.M.F., Bourgeois, F., 1998. The CSIRO Hopkinson bar facility for large diameter particle breakage. Miner. Eng. 11 (9), 861-869.

Gaudin, A.-M., 1939. Principles of Mineral Dressing. McGraw-Hill Book Company, Inc.

Jeschke, H., Poppy, W., Schubert, W., 2006. Concrete comminution in impact crushersexperiments and simulations. Miner. Process. 47 (6), 4-21.

Khanal, M., Schubert, W., Tomas, J., 2005. Experiment and simulation of breakage of particle compounds under compressive loading. Part. Sci. Technol. 23 (4), 387-394.

King, R.P., Bourgeois, F., 1993. A new conceptual model for ball milling. Proceedings of the XVIII International Mineral Processing Congress. 1, pp. 81-86 (Australia).

Lippiatt, N., Bourgeois, F., 2012. Investigation of microwave-assisted concrete recycling using single-particle testing. Miner. Eng. 31, 71-81.

Lippiatt, N., Bourgeois, F., 2014. Recycling-oriented investigation of local porosity changes in microwave heated-concrete. KONA Powder Part. J. 31, 247-264.

Lynch, A.J., 1977. Mineral Crushing and Grinding Circuits-Their Simulation, Optimisation, Design and Control. Elsevier Scientific Publishing Company, Amsterdam-Oxford-New York (1977).

Mehta, P., Monteiro, P., 2001. Concrete: microstructure, properties and materials, McGraw-Hill Professional (2001).

Middlemiss, S., 2007. Surface damage effects in single particle comminution. Int. J. Miner. Process. 84, 207-220.

Middlemiss, S., King, R.P., 1996. Microscale fracture measurements with application to comminution. Int. J. Miner. Process. 44-45, 43-58.

Powell, M.S., Govender, I., McBride, A.T., 2008. Applying DEM outputs to the unified comminution model. Miner. Eng. 21, 744-750.

Qian, Z., Schlange, E., 2013. Lattice modeling of fracture processes in numerical concrete with irregular shape aggregates. Proceeding of the 8th International Conference on Fracture Mechanics of Concrete and Concrete Structures, pp. 1539-1545 (March 10-14, 2013/Toledo - Spain).

Schönert, K., Marktscheffel, 1986. Liberation of composite particles by single particle compression, shear and impact loading. Proc. 6th Eur. Symp. Commin., Nümberg, p. 29.

Tavares, L.M., King, R.P., 1998. Single-particle fracture under impact loading. Int. J. Miner Process. 54, 1-28.

Weatherley, D.K., 2013. Numerical investigation relating to lithology and mechanical properties of rock. In: Kwade, A., Breitung-Faes, S., Steiner, D. (Eds.), Proceedings of the 13th European Symposium on Comminution \& Classification, pp. 221-224 (9th-12th September 2013, Braunschweig, Germany).

Weerasekara, N.S., Powell, M.S., Cleary, P.W., Tavares, L.M., Evertsson, M., Morrison, R.D. Quist, J., Carvalho, R.M., 2013. The contribution of DEM to the science of comminution. Powder Technol. 248, 3-24.

William, K., Rhee, I., Xi, Y., 2005. Thermal degradation in heterogeneous concrete materials. J. Mater. Civ. Eng. ASCE 17 (3), 276-285.

Yashima, S., Kanda, Y., Sano, S., 1987. Relationships between particle size and fracture energy or impact velocity required to fracture as estimated from single particle crushing. Powder Technol. 51, 277-282. 